\title{
Single-cell systems neuroscience: A growing frontier in mental illness
}

\author{
SEAN J. O'SULLIVAN ${ }^{1,2, *}$ \\ ${ }^{1}$ Daniel Baugh Institute for Functional Genomics and Computational Biology, Department of Pathology, Anatomy and Cell Biology, Thomas Jefferson \\ University, Philadelphia, USA \\ ${ }^{2}$ Brain Stimulation Laboratory, Stanford University, Stanford, USA
}

Key words: Systems biology, Systems neuroscience, Single-cell psychiatry, Psychopathology

\begin{abstract}
The development of effective treatments for psychiatric disease has been disappointing in recent decades given the advancements in neuroscience. Moreover, rising rates of mental illness such as addiction and depression compel scientists and physicians to discover novel and creative solutions. One such approach that has proven effective is systems neuroscience: A focus on networks as opposed to mechanism. Further, investigation at the single-cell and circuit level is likely to be fruitful in such endeavors as this resolution describes the functional psychopathology that allows for intervention.
\end{abstract}

\section{Introduction}

Rates and severity of mental illness in the United States have risen over the past decade ("Key Substance Use and Mental Health Indicators in the United States: Results from the 2019 National Survey on Drug Use and Health" 2020; Merikangas et al., 2010; NIMH, 2020). Infection by the novel coronavirus and indirect effects of the pandemic on mental health suggest this increase is likely to continue and possibly accelerate (Vindegaard and Benros, 2020; Taquet et al., 2020). In addition to individual suffering, the socioeconomic and politico-cultural consequences of this rise in mental illness compels investigators and clinicians alike towards realizing novel understandings and treatments as canonical psychiatric therapies are often burdened by low efficacy, high side-effect profiles, and ballooning costs (Holmes et al., 2014; Holmes et al., 2018; Ford and Young, 2021).

Neuropsychopathology studies, like virtually all biological investigation, are performed under the guise that the data from the experiments will eventually contribute to clinical treatment. Though neuroscientific understanding has developed rapidly in recent decades, novel psychiatric therapies remain elusive (Holmes et al., 2014; Holmes et al., 2018; Ford and Young, 2021). Even the recent psychedelic revolution is a rediscovery of medications from half a

${ }^{*}$ Address correspondence to: Sean J. O’Sullivan, sean.o’sullivan@jefferson.edu

Received: 30 May 2021; Accepted: 19 July 2021 century prior (Johnson et al., 2019). The demand for efficacious clinical treatments with minimal side effects, however, remains high which galvanizes investigator creativity raising the attractiveness of newer approaches to neuropsychopathology such as systems biology.

Systems biology distinguishes itself from hypothesisdriven research in that understanding networks is the primary objective as opposed to elucidating mechanisms (Ideker et al., 2001; Geschwind and Konopka, 2009). This difference shifts experimentation towards highthroughput measures at multiple levels of biological systems with an emphasis on physiological context and mathematical modeling. Experimental designs with such broad parameters that do not isolate individual variables had been previously untenable because of cost and reliability; However, advances in biotechnologies and computer science have diminished such barriers (Geschwind and Konopka, 2009). Moreover, this approach emphasizes network states and thus avoids the challenges of determining causality that are inherent in psychiatry (Stojanov et al., 2011).

Indeed, the dam of systems neuroscience has burst, and datasets filled with pearls are flooding the field. At the level of organ systems, visceral feedback to central centers has been shown to have profound effects on mental health (Critchley and Harrison, 2013). The gut-brain connection has proven particularly powerful as intestinal microflora composition consistently demonstrate behavioral influence (Sylvia and Demas, 2018). Moreover, developments in imaging have allowed investigators to map neural modules 
and their relationships to psychopathology. This has led to the development of neuromodulatory interventions such as transcranial magnetic stimulation that is now Food and Drug Administration (FDA) approved for treatment-resistant depression and obsessive-compulsive disorder (Horvath et al., 2010; Voelker, 2018). At the molecular level, genetic sequencing and transcriptome measures are yielding datadriven models of cellular signaling networks that challenge prior notions of neuronal phenotypes (Park et al., 2014).

The emergence of single cell studies is particularly fruitful in this new frontier (Callaway, 2005). Nervous tissue contains many kinds of cells ranging from various types of neurons to astrocytes, oligodendrocytes, microglia, endothelial cells, and so on. The firing of any individual neuron-which, en masse, is still believed to be the modus for perception, thought, and behavior-is known to be influenced by the context in which that neuron finds itself (Pereira and Furlan, 2010; Callaway, 2005). Anatomic localization within a network, the neighboring neurons, synaptic and non-synaptic inputs, local paracrine signaling and glial influences in combination determine the role of that neuron within the network and its output. The complexity of this dance may never be fully understood even with quantum computers (Schiffer, 2019), but singlecell systems neuroscience is beginning to point us towards the prominent concepts at this level that elude mechanistic molecular studies and tissue-level or higher approaches. That is, single-cell studies are in a Goldilocks zone of biological levels for neuropsychopathology investigation-as signaling networks and circuits are deciphered at this level, knowledge of normal physiology and pathophysiology in mental illness is likely to translate to clinical treatments (Ford and Young, 2021).
An example of this approach is our recent work measuring the effects of opioid withdrawal on gene expression in single brain cells in the central nucleus of the amygdala (CeA) of rats (O'Sullivan et al., 2019). We combined single-cell laser capture microdissection with microfluidic RT-qPCR to measure a selected transcriptome of neurons, microglia, and astrocytes with anatomic specificity (O'Sullivan et al., 2020). The pre-experimental hypothesis, based on our prior hypothesis-generating work (Freeman et al., 2012a; Freeman et al., 2013; Freeman et al., 2012b), was that neuroinflammation, likely originating from microglia, would be present 24 hours following acute naltrexone-precipitated opioid withdrawal. Rather, we found a striking transcriptional response in astrocytes. A review of the literature contextualized this finding and led us to a profound conjecture-that neuroinflammation mediated by astrocytes in the CeA contributes to the negative emotion characteristic of opioid withdrawal and that this neuroinflammation increases the negative reinforcement of withdrawal (O'Sullivan and Schwaber, 2021). Moreover, this work translates to other levels of biological systems including molecular signaling, organ systems, and behavior, and has implications for the mechanisms of action of new therapies.

Astrocytes had the most perturbed transcriptome of the assayed cell types, but neurons and microglia also demonstrated a significant increase in the expression of tumor necrosis factor alpha (TNF- $\alpha$ ) (Fig. 1). This finding was validated with Western blot and immunofluorescence and suggests that this cytokine is a primary molecular signal in the negative emotional sequelae of opioid withdrawal. TNF- $\alpha$ has been shown to lower neuronal excitation threshold resulting in an increased probability of actional potential firing (Schäfers and Sorkin, 2008; Vezzani and Viviani, 2015). Taken together, these findings suggest TNF- $a$
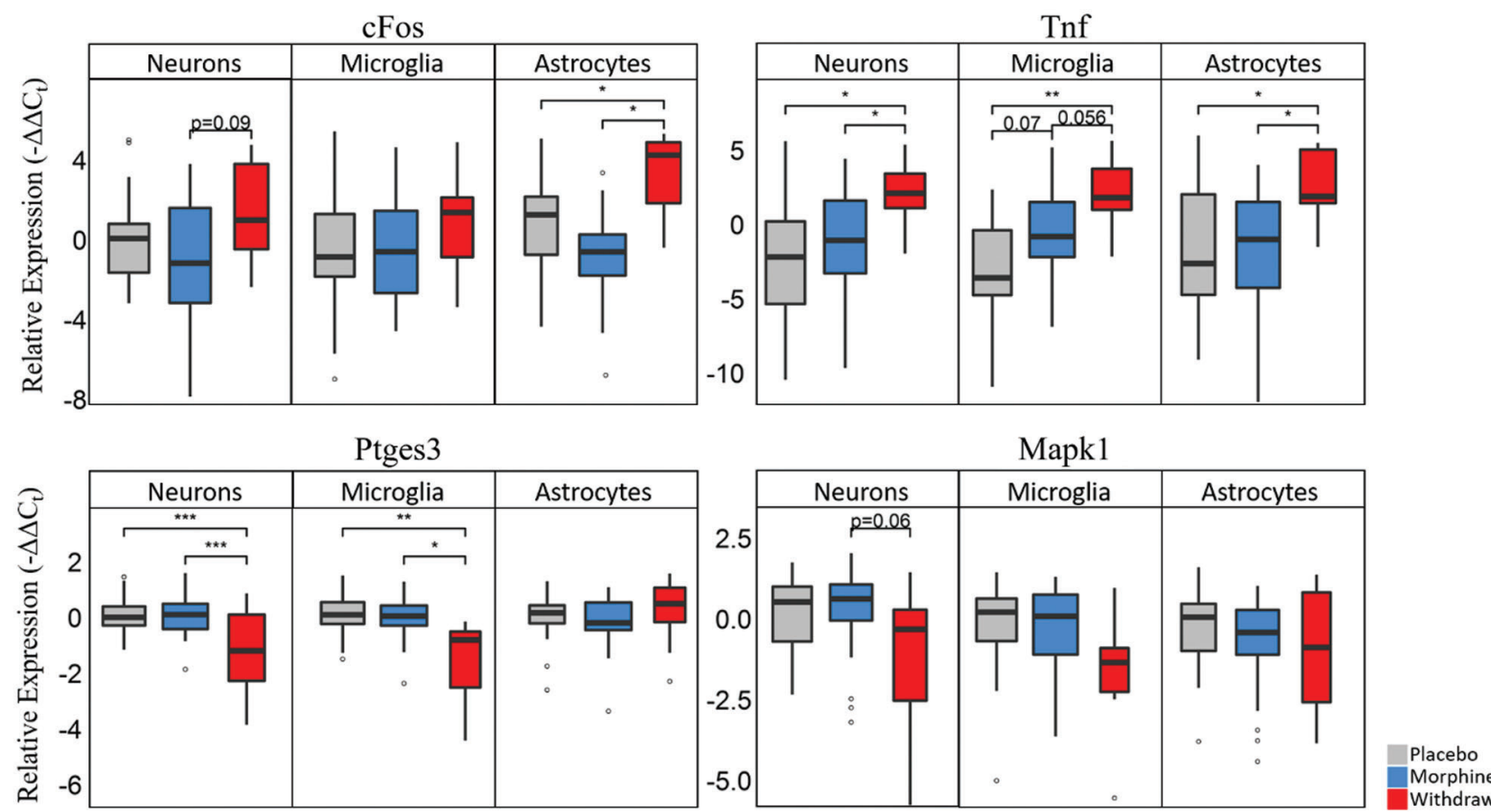

FIGURE 1. Boxplots of select genes demonstrating significant differential gene expression.

Statistics were calculated using nested ANOVA $\left({ }^{*} p<0.05,{ }^{* *} p<0.01,{ }^{* * *} p<0.0001 \mathrm{n}=4\right.$ animals for all treatments). Originally published in O'Sullivan et al. (2019). 
paracrine signaling, likely mediated by astrocytes, in the CeA during opioid withdrawal may lead to hyperactivity that contributes to negative emotion and reinforcement of drug taking.

At the systems level, the CeA has strong bidirectional connections to the nucleus tractus solitarius (NTS)- the primary viscerosensory nucleus in the central nervous system (Schwaber et al., 1982). This neuroanatomy suggests that the emotions experienced secondary to limbic activity are substantially influenced by peripheral feedback; an insight that has been shown convincingly (Maniscalco and Rinaman, 2018). Additionally, we found that gut microflora demonstrated remarkable perturbation in opioid withdrawal (Fig. 2) (O'Sullivan et al., 2019). This finding was correlated with astrocyte activation in the amygdala suggesting these two observations may be linked via interoceptive vagal afferents and the NTS.

These single-cell findings translate to the behavioral level as well. We conjecture that these connections form a visceralemotional neuraxis in which peripheral perturbation sensed by vagal afferents is transmitted to the amygdala forming an interoceptive antireward pathway (O'Sullivan and Schwaber, 2021). This antireward circuit is inhibited by drug use and stimulated by substance withdrawal. We hypothesize that the mesolimbic dopamine reward pathway functions in parallel to this circuit with inverse activity consistent with the opponent-process model (Pierce and Kumaresan, 2006; Wise, 2008; Solomon and Corbit, 1974). These reward and antireward pathways combine to provide positive and negative motivators that reinforce behavior. Compulsive drug-seeking and taking, then, is an imbalance in this behavioral paradigm that is influenced at the molecular level.

This model informs future studies and the mechanism of action of recent therapies. Astrocyte-mediated inflammation in CeA has been demonstrated to provoke anxiety-like behavior, (Yang et al., 2016) but the temporal dynamics of this process in opioid withdrawal and the contribution of visceral feedback remain unknown. Subdiaphragmatic vagotomy studies in rat methamphetamine self-administration suggest this circuit has an important influence on addiction (Everett et al., 2021), but further investigation is warranted.
A

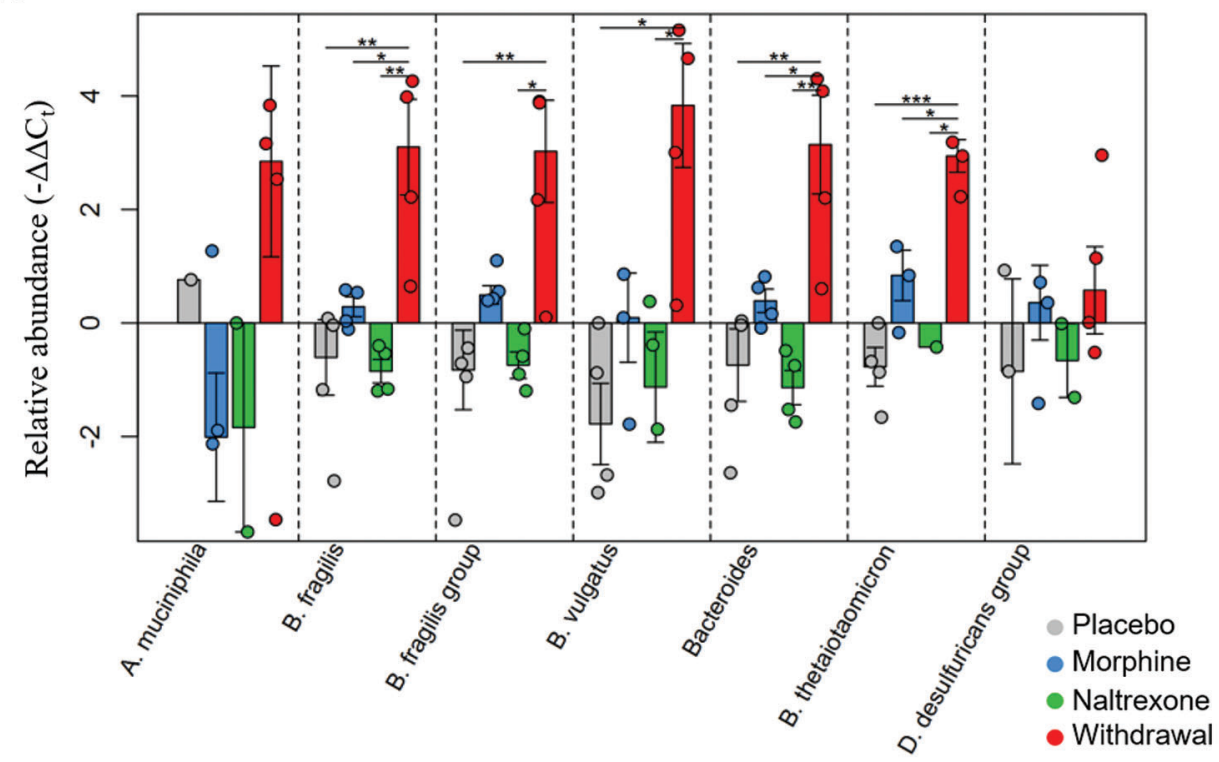

B

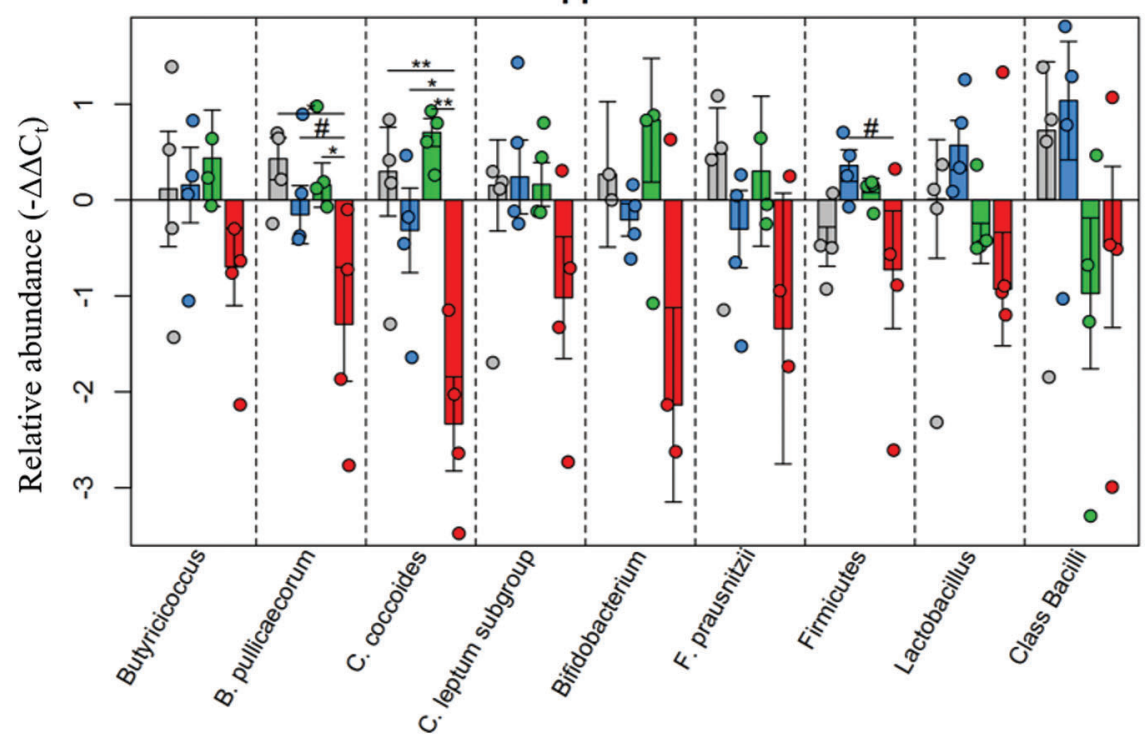

FIGURE 2. Relative abundance of gut microflora from rat cohort. Barplots display relative abundance of bacterial species $\left(-\Delta \Delta \mathrm{C}_{\mathrm{t}}\right.$ values). ${ }^{\#} p<$ $0.1,{ }^{*} p<0.05,{ }^{* *} p<0.008,{ }^{* *} p=$ 0.0009 ; two-way ANOVA $\mathrm{n}=4$ animals for each treatment. Originally published in O'Sullivan et al. (2019). 
Moreover, anti-neuroinflammatory interventions such as ibudilast have shown promise in treating addiction to multiple substances in clinical trials (Cooper et al., 2016; Heinzerling et al., 2020; Worley et al., 2016; Metz et al., 2017; Comer and Johnson, 2013). It reduced the physical and emotional symptoms of opioid withdrawal syndrome. Furthermore, there is evidence that ibudilast's mechanism of action may be a peripheral decrease in inflammation consistent with our model (Li et al., 2020). Indeed, peripheral stimulation for opioid withdrawal syndrome has recently been approved by the FDA (Miranda and Taca, 2018). The BRIDGE device provides peripheral simulation to auricular cranial nerves during opioid withdrawal and investigators have shown that this input decreases neuronal firing in the amygdala via the NTS. This device likely treats opioid withdrawal symptoms by decreasing CeA neuronal activity via peripheral stimulation consistent with our findings and model (O'Sullivan et al., 2019; O'Sullivan and Schwaber, 2021; O'Sullivan et al., 2021).

In conclusion, single-cell studies are situated at a unique level of biological analysis that is particularly fruitful for developing much needed psychiatric treatments. Single-cell experiments with a network focus not only inform our understanding of neural circuits, but also paracrine signaling by a spectrum of cell types and provide context for organ systems and behavioral understandings. The models generated from such studies require validation by more mechanistic approaches, but these models and the hypotheses they suggest can provide insight into the mechanisms of action of novel therapies.

Acknowledgement: SJO would like to acknowledge James S. Schwaber, Ph.D. for his mentorship in single-cell and systems neuroscience.

Availability of Data and Materials: No data or materials were generated from this study.

Author Contribution: SJO confirms sole responsibility for the manuscript writing and viewpoint.

Ethics Approval: There were no experiments involving humans, animals, or plants in this study.

Funding Statement: The author received no specific funding for this study.

Conflicts of Interest: The author declares that they have no conflicts of interest to report regarding the present study.

\section{References}

Callaway EM (2005). A molecular and genetic arsenal for systems neuroscience. Trends in Neurosciences 28: 196-201. DOI 10.1016/j.tins.2005.01.007.

Comer S, Johnson K (2013). A drug candidate for improving opioid analgesia and attenuating dependence and tolerance: An exploratory trial of ibudilast in morphine withdrawal and analgesia in heroin addicts (P07.183). Neurology 80: P07.183. http://www.neurology.org/cgi/content/meeting_abstract/80/1_ MeetingAbstracts/P07.183.
Cooper ZD, Johnson KW, Pavlicova M, Glass A, Vosburg SK et al. (2016). The effects of ibudilast, a glial activation inhibitor, on opioid withdrawal symptoms in opioid-dependent volunteers. Addiction Biology 21: 895-903. DOI 10.1111/ adb.12261.

Critchley HD, Harrison NA (2013). Visceral influences on brain and behavior. Neuron 77: 624-638. DOI 10.1016/j.neuron.2013.02.008.

Everett NA, Turner AJ, Costa PA, Baracz SJ, Cornish JL (2021). The vagus nerve mediates the suppressing effects of peripherally administered oxytocin on methamphetamine selfadministration and seeking in Rats. Neuropsychopharmacology 46: 297-304. DOI 10.1038/s41386-020-0719-7.

Ford CL, Young LJ (2021). Translational opportunities for circuitbased social neuroscience: Advancing 21st century psychiatry. Current Opinion in Neurobiology 68: 1-8. DOI 10.1016/j.conb.2020.11.007.

Freeman K, Brureau A, Vadigepalli R, Staehle MM, Brureau MM et al. (2012a). Temporal changes in innate immune signals in a rat model of alcohol withdrawal in emotional and cardiorespiratory homeostatic nuclei. Journal of Neuroinflammation 9: S13. DOI 10.1186/1742-2094-9-97.

Freeman K, Staehle MM, Gumus H, Vadigepalli R, Gonye GE et al. (2012b). Rapid temporal changes in the expression of a set of neuromodulatory genes during alcohol withdrawal in the dorsal vagal complex: Molecular evidence of homeostatic disturbance. Alcoholism: Clinical and Experimental Research 36: 1688-1700. DOI 10.1111/j.1530-0277.2012.01791.x.

Freeman K, Staehle MM, Vadigepalli R, Gonye GE, Ogunnaike BA et al. (2013). Coordinated dynamic gene expression changes in the central nucleus of the amygdala during alcohol withdrawal. Alcoholism: Clinical and Experimental Research 37: E88-E100. DOI 10.1111/j.1530-0277.2012.01910.x.

Geschwind DH, Konopka G (2009). Neuroscience in the era of functional genomics and systems biology. Nature. Nature Publishing Group. https://doi.org/10.1038/nature0853.

Heinzerling KG, Briones $\mathrm{M}$, Thames D, Hinkin $\mathrm{CH}$, Zhu $\mathrm{T}$ et al. (2020). Randomized, placebo-controlled trial of targeting neuroinflammation with ibudilast to treat methamphetamine use disorder. Journal of Neuroimmune Pharmacology 15: 238-248. DOI 10.1007/s11481-019-09883-w.

Holmes EA, Craske MG, Graybiel AM (2014). Psychological treatments: A call for mental-health science. Nature. Nature Publishing Group. https://doi.org/10.1038/511287a.

Holmes EA, Ghaderi A, Harmer CJ, Ramchandani PG, Cuijpers P et al. (2018). The lancet psychiatry commission on psychological treatments research in tomorrow's science. Lancet Psychiatry 5: 237-286. DOI 10.1016/S2215-0366(17)30513-8.

Horvath JC, Mathews J, Demitrack MA, Pascual-Leone A (2010). The NeuroStar TMS device: conducting the FDA approved protocol for treatment of depression. Journal of Visualized Experiments 45: 2345. DOI 10.3791/2345.

Ideker T, Galitski T, Hood L (2001). A new approach to decoding life: Systems biology. Annual Review of Genomics and Human Genetics. https://doi.org/10.1146/annurev.genom.2.1.343.

Johnson MW, Hendricks S, Barrett S, Griffiths RR (2019). Classic psychedelics: An integrative review of epidemiology, therapeutics, mystical experience, and brain network function. Pharmacology \& Therapeutics 197: 83-102. DOI 10.1016/j.pharmthera.2018.11.010.

Key Substance Use and Mental Health Indicators in the United States: Results from the 2019 National Survey on Drug Use and Health (2020). Rockville, MD. https://www.samhsa.gov/data/. 
Li MJ, Briones MS, Heinzerling KG, Kalmin MM, Shoptaw SJ (2020). Ibudilast attenuates peripheral inflammatory effects of methamphetamine in patients with methamphetamine use disorder. Drug and Alcohol Dependence 206: 107776. DOI 10.1016/j.drugalcdep.2019.107776.

Maniscalco JW, Rinaman L (2018). Vagal interoceptive modulation of motivated behavior. Physiology 33: 151-167. DOI 10.1152/physiol.00036.2017.

Merikangas KR, He JP, Burstein M, Swanson SA, Avenevoli S et al. (2010). Lifetime prevalence of mental disorders in U.S. adolescents: Results from the national comorbidity survey replication-adolescent supplement (NCS-A). Journal of the American Academy of Child \& Adolescent Psychiatry 49: 980989. DOI 10.1016/j.jaac.2010.05.017.

Metz VE, Jones JD, Manubay J, Sullivan MA, Mogali S et al. (2017). Effects of ibudilast on the subjective, reinforcing, and analgesic effects of oxycodone in recently detoxified adults with opioid dependence. Neuropsychopharmacology 42: 1825-1832. DOI 10.1038/npp.2017.70.

Miranda A, Taca A (2018). Neuromodulation with percutaneous electrical nerve field stimulation is associated with reduction in signs and symptoms of opioid withdrawal: A multisite, retrospective assessment. American Journal of Drug and Alcohol Abuse 44: 56-63. DOI 10.1080/ 00952990.2017.1295459.

NIMH (2020). Prevalence of Serious Mental Illness (SMI). https:// doi.org/10.1016/j.jaac.2010.05.017.

O’Sullivan SJ, McIntosh-Clarke D, Park J, Vadigepalli R, Schwaber JS (2021). Single neuronal and glial gene expression in the nucleus tractus solitarius in an alcohol withdrawal time series reveals novel cellular phenotypes and networks. Research Square. https://doi.org/10. 21203/RS.3.RS-452767/V1.

O'Sullivan SJ, Malahias E, Park J, Srivastava A, Reyes BAS et al. (2019). Single-cell glia and neuron gene expression in the central amygdala in opioid withdrawal suggests inflammation with correlated gut dysbiosis. Frontiers in Neuroscience 13: 113. DOI 10.3389/fnins.2019.00665.

O’Sullivan SJ, Reyes BAS, Vadigepalli R, Van Bockstaele EJ, Schwaber JS (2020). Combining laser capture microdissection and microfluidic qPCR to analyze transcriptional profiles of single cells: A systems biology approach to opioid dependence. Journal of Visualized Experiments 157: e60612. DOI $10.3791 / 60612$.

O'Sullivan SJ, Schwaber JS (2021). Similarities in alcohol and opioid withdrawal syndromes suggest common negative reinforcement mechanisms involving the interoceptive antireward pathway. Neuroscience \& Biobehavioral Reviews 125: 355-364. DOI 10.1016/j.neubiorev.2021.02.033.

Park J, Brureau A, Kernan K, Starks A, Gulati S et al. (2014). Inputs drive cell phenotype variability. Genome Research 24: 930941. DOI 10.1101/gr.161802.113.

Pereira A, Furlan FA (2010). Astrocytes and human cognition: Modeling information integration and modulation of neuronal activity. Progress in Neurobiology. Pergamon 92: 405-420. DOI 10.1016/j.pneurobio.2010.07.001.

Pierce RC, Kumaresan V (2006). The mesolimbic dopamine system: The final common pathway for the reinforcing effect of drugs of abuse? Neuroscience \& Biobehavioral Reviews 30: 215-238. DOI 10.1016/j.neubiorev.2005.04.016.

Schäfers M, Sorkin L (2008). Effect of cytokines on neuronal excitability. Neuroscience Letters 437: 188-193. DOI 10.1016/j.neulet.2008.03.052.

Schiffer F (2019). The physical nature of subjective experience and its interaction with the brain. Medical Hypotheses 125: 57-69. DOI 10.1016/j.mehy.2019.02.011.

Schwaber JS, Kapp BS, Higgins GA, Rapp PR (1982). Amygdaloid and basal forebrain direct connections with the nucleus of the solitary tract and the dorsal motor nucleus. Journal of Neuroscience 2: 1424-1438. DOI 10.1523/JNEUROSCI.0210-01424.1982.

Solomon RL, Corbit JD (1974). An opponent-process theory of motivation: I. Temporal dynamics of affect. Psychological Review 81: 119-145. DOI 10.1037/h0036128.

Stojanov D, Korf J, de Jonge P, Popov G (2011). The possibility of evidence-based psychiatry: Depression as a case. Clinical Epigenetics 2: 7-15. DOI 10.1007/s13148-010-0014-2.

Sylvia KE, Demas GE (2018). A gut feeling: Microbiome-brainimmune interactions modulate social and affective behaviors. Hormones and Behavior. Academic Press Inc. https://doi.org/10.1016/j.yhbeh.2018.02.001.

Taquet M, Luciano S, Geddes JR, Harrison PJ (2020). Bidirectional associations between COVID-19 and psychiatric disorder: Retrospective cohort studies of 62354 COVID-19 cases in the USA. Lancet Psychiatry 8: 130-140. DOI 10.1016/S22150366(20)30462-4.

Vezzani A, Viviani B (2015). Neuromodulatory properties of inflammatory cytokines and their impact on neuronal excitability. Neuropharmacology 96: 70-82. DOI 10.1016/j. neuropharm.2014.10.027.

Vindegaard N, Benros ME (2020). COVID-19 Pandemic and mental health consequences: Systematic review of the current evidence. Brain, Behavior, and Immunity. Academic Press Inc. https://doi.org/10.1016/j.bbi.2020.05.048.

Voelker R (2018). Brain stimulation approved for obsessive-compulsive disorder. JAMA 320: 1098. DOI 10.1001/jama.2018.13301.

Wise RA (2008). Dopamine and reward: The anhedonia hypothesis 30 years on. Neurotoxicity Research 14: 169-183. DOI 10.1007/BF03033808.

Worley MJ, Heinzerling KG, Roche DJO, Shoptaw S, Shoptaw S (2016). Ibudilast attenuates subjective effects of methamphetamine in a placebo-controlled inpatient study. Drug and Alcohol Dependence 162: 245-250. DOI 10.1016/j.drugalcdep.2016.02.036.

Yang L, Wang M, Guo YY, Sun T, Li YJ et al. (2016). Systemic inflammation induces anxiety disorder through CXCL12/ CXCR4 pathway. Brain, Behavior, and Immunity 56: $352-$ 362. DOI 10.1016/j.bbi.2016.03.001. 